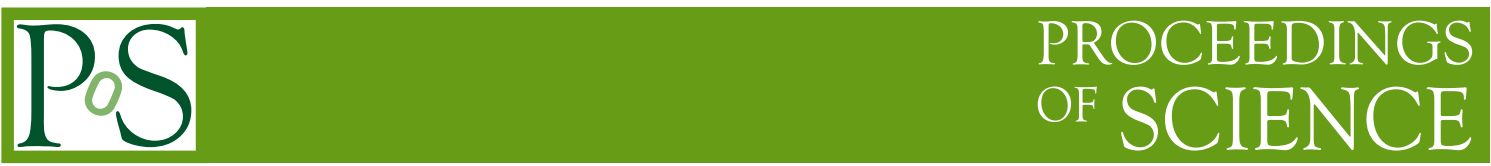

\title{
Review on Dark Matter Tools
}

\author{
Chiara Arina ${ }^{a, *}$ \\ ${ }^{a}$ Centre for Cosmology, Particle Physics and Phenomenology (CP3), IRMP, UCLouvain, \\ Chemin du Cyclotron 2, 1348 Louvain-la-Neuve, Belgium
}

E-mail: chiara.arina@uclouvain. be

Whilst the need for dark matter was established almost a century ago, only its gravitational interaction has been confirmed so far, allowing for plethora of models for dark matter. The Weakly Interacting Massive Particles (WIMPs) category has received by far the biggest attention, however despite the enormous experimental efforts, these particles remain elusive. The attention of the community has hence moved on to investigate the dark matter landscape over a much larger number of models with varying degrees of resemblances and differences in their predictions. This calls for the need to organise the various facets of dark matter models and their signatures, in order to maximise the experimental sensitivity and to select the models which are compatible with existing data. In this paper, I provide a short review of the most widespread public codes capable of computing dark matter observables. In particular, I discuss what is the status of each numerical tool in terms of: (i) capturing the WIMP phenomenology and (ii) accounting for new trend dark sector models that might be weakly coupled to ordinary matter and/or be strongly self-interacting. This short review has the aim of guiding the user towards selecting the best suited public code to confront his/her model with the largest variety of theoretical predictions and experimental data in order to determine the parameter space consistent with observations for his/her favourite dark matter model.

Tools for High Energy Physics and Cosmology - TOOLS2020

2-6 November, 2020

Institut de Physique des 2 Infinis (IP2I), Lyon, France

\footnotetext{
${ }^{*}$ Speaker
} 


\section{Introduction}

The quest for dark matter has started almost a century ago, however at present very little is known about its nature. Its presence is strongly required for instance by the cosmological model $\Lambda \mathrm{CDM}$ [1], structure formation [2-4], galaxy rotation curves, see i.e. [5, 6]. The astrophysical and cosmological hints can however only probe the gravitational interaction of dark matter and provide no insights in other types of interactions, in particular with the Standard Model (SM) particles.

Under the assumption that dark matter particles do interact with SM particles, the most studied candidates so far are the so-called Weakly Interacting Massive Particles (WIMPs). These particles feature interaction strength of the order of the weak force and consequently achieve naturally the correct relic density in the $\mathrm{GeV}$ and $\mathrm{TeV}$ mass range. Besides the fact that they arise in many models beyond the SM, such as supersymmetry (SUSY) or extra-dimension, see e.g. [6], they result in a variety of signals at accessible energy scales. A multitude of experimental approaches has been undertaken to detect WIMPs, ranging from dark matter searches using underground detectors (direct detection), to observations of gamma rays, cosmic rays and neutrinos in astrophysical environments (indirect detection), and searches for missing energy signals at colliders (production). Yet, despite the enormous experimental effort, WIMPs remain elusive. Even though they are not excluded, see e.g. [7], new directions are explored, such as dark hidden sectors, Feebly Interacting Massive Particles (FIMPs) [8], sub-GeV dark matter candidates [9]. For instance, these latter imply that dark matter direct detection arises via electronic recoils (instead of the standard nuclear recoils), while the former may imply very weakly coupled particles to the SM but strongly self-interacting and decoupling from the thermal bath with a temperature different than to one of the SM particles. The FIMPs encompass dark matter candidates in all the mass range between sub-GeV to TeV. These particles are feebly coupled to the SM and for this reason not in thermal equilibrium in the early universe, but produced by the freeze-in mechanism instead, see $[10,11]$ for details. These are only few examples to make the point that these new avenues require new theoretical frameworks for the predictions and consequently call for new numerical routines to compare those predictions with the experimental results in the spirit of global fits.

From the software point of view, this last decade is characterised by constant efforts to improve the public codes for dark matter observables along three main directions: (i) cover all WIMP phenomenology and improve its accuracy with the inclusion of next-to-leading order (NLO) corrections; (ii) include the new phenomenology arising in the new dark matter paradigm and (iii) make the public code portable/well linked with other available software. This should help the user to perform global predictions and sampling of the parameter space, simultaneously taking into account constraints from collider and different dark matter probes. Even though a bit aside from the scope of this review, point (iii) is of the uttermost importance and few comments are in order, since it is developed in several directions. On the one hand, FeynRules [12], SARAH [13] and LanHEP [14] allow the user to implement his/her dark matter model at the level of the Lagrangian, and produce output files for several high energy public tools, including MicrOMEGAs and MADDM for computing dark matter observables. Several dark matter models are already publicly available in the FeynRules database ${ }^{1}$, such as the so-called $s$ - and $t$-channel simplified models ${ }^{2}$ used at the LHC,

$\mathbf{1}_{\text {https://feynrules.irmp.ucl.ac.be/wiki/ModelDatabaseMainPage }}$

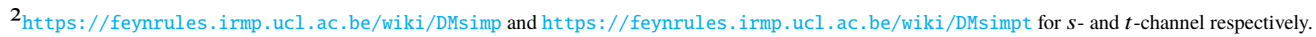


see [15-19]. From the same model Lagrangian, FeynRules provides output files for computing collider predictions with MG5_AMC [20] (UFO format [21], the same used in MADDM) and to apply collider bounds with the recasting tool MADANALYsis 5 [22]. Alternatively, from MicrOMEGAs it is straightforward to compute collider exclusion bounds in the simplified model framework by using the SModelS tool [23]. These chains of tools are pretty well established and have led to numerous publications, see e.g. [24-27]. On the other hand, the GAMBIT collaboration [28] provides various modules for dark matter observables [29], cosmology [30], sampling [31], to perform consistently global analysis of dark matter models, see e.g. [32-35].

In this review I will focus specifically on dark matter tools and consider only the four major public and generic codes: SuperIso Relic ${ }^{3}$ [36], DARkSUSY ${ }^{4}$ [37], MicrOMEGAs ${ }^{5}$ [38] and MADDM $^{6}[39,40]$. There are many more specific dark matter tools available on the market, which I will mention whenever relevant. In section 2, I will briefly review the current status of these numerical tools in relation with the coverage of the WIMP phenomenology. Subsequently in section 3, I will discuss the most salient features that go beyond the WIMP scenario for each tool separately. My concluding remarks are discussed in section 4. This short paper is not meant to give an exhaustive description of the new avenues towards dark sectors nor an in-depth description of the public codes, for which I refer to the documentation provided by the authors of the software. This review is rather meant to highlight features that distinguish the different numerical tools, in such a way to guide the user towards the most suitable code for his/her purpose.

\section{WIMPs: where do the tools stand so far?}

The development of public generic tools has started already at the beginning of the 21 st century: MicrOMEGAs [41] is the first code being developed, followed by DARKSUSY [42], SuPERIso ReLIC [43] and then subsequently by MADDM, which is by far the more recent one [44]. As all codes are on the market from a few years now, they all cover the necessary basics of the WIMP phenomenology, to various extent.

Relic density WIMPs achieve the correct relic density via the freeze-out mechanism. The dark matter particles have interactions of the order of the electroweak force, which keep them in chemical (and kinetic) equilibrium in the thermal bath enough to decouple when they are non-relativistic and at the photon temperature for which the thermally averaged cross-section $\langle\sigma v\rangle$ is around $3 \times 10^{-26} \mathrm{~cm}^{3} / \mathrm{s}$ (for the $s$-wave annihilation at low relative velocity $v$ ). This ensures that the relic abundance is close to $\Omega h^{2}=0.12$, value measured by Planck [1]. Departing from this simple case, dark matter particles can feature a $\langle\sigma v\rangle$ which varies rapidly with energy close to resonances or to thresholds of new annihilation channels, or the model can have coannihilation. The latter arises when there are new particles beyond the SM ones, which are odd under the dark group similarly to the dark matter particle, and which are close in mass to the dark matter particles (namely 10\% $15 \%$ in mass splitting at most). These heavier particles are present in the thermal bath at the time of dark matter freeze-out and their annihilations contribute to it. To account then for all annihilation

\footnotetext{
$3_{\text {http://superiso.in2p3.fr/relic/ }}$

$\mathbf{4}_{\text {https: //darksusy . hepforge.org/ }}$

$5_{\text {https://lapth.cnrs.fr/micromegas/ }}$

$\mathbf{6}_{\text {https://launchpad.net/maddm }}$
} 
and coannihilation processes it is customary to define an effective thermally averaged cross-section $\langle\sigma v\rangle_{\mathrm{eff}}$, see i.e. [45, 46].

All public codes provide the exact relativistic single-integral formula for $\langle\sigma v\rangle_{\mathrm{eff}}$ and solve the corresponding Boltzmann equation to find the dark matter yield at present time. More specifically, SuPERIso ReLIC has included analytically all the hundreds of diagrams relevant for neutralino annihilation and coannihilation in supersymmetric scenarios such as the MSSM. This is similar to the case of DARKSUSY which was born for supersymmetry, even though the user can directly provide $\langle\sigma v\rangle$ for his/her model in order to compute $\Omega h^{2}$, which is in addition determined by using the most precise treatment described in [47]. MICROMEGAs and MADDM rely on the on-the-fly computation of all matrix elements by CALCHEP [48] and MG5_AMC respectively, including coannihilation processes; they additionally handle the case of multi-component dark matter. Furthermore, the user can improve $\langle\sigma v\rangle$, such as with NLO terms, by editing a simple predefined function within MicROMEGAs. There are indeed works that have shown that NLO corrections are relevant, for instance when the annihilation cross-section is suppressed by $d$-wave $\left(v^{4}\right)$ or in supersymmetric models, see e.g. $[49,50]$. In the case of supersymmetry, few specific packages have been developed, such as DM@NLO [51], which are typically compatible with MicrOMEGAs. DARKSUSY allows for the computation of the kinetic decoupling of dark matter particles, which happens much after they have frozen-out (chemical decoupling). This is phenomenologically relevant as it firstly includes higher moments in the Boltzmann equation, which can considerably change the relic density prediction in some corners of the model parameter space, and secondly sets a relevant scale for the formation of small-scale structures with observational consequences in indirect detection signals, see [52, 53].

Direct detection Standard dark matter direct searches measure the recoil energy of nuclei hit by dark matter particles crossing (large) detectors deep underground. The typical recoil energy is at the $\mathrm{keV}$ energy scale, as the dark matter particles are non-relativistic, have a velocity of about $220 \mathrm{~km} / \mathrm{s}$ which lead to a momentum transfer in the scattering of the order of $\mathrm{MeV}$, see e.g. [54-57] for the generalities. Because of this small velocity, typically all terms proportional to the momentum transfer and to the velocity, are neglected and only the leading spin-independent and spin-dependent interaction terms are kept. This is the approach followed by MicrOMEGAs and MADDM to compute the elastic cross-section for WIMP-nuclei scattering at both leading order and NLO in the case of the former code. The NLO corrections in general take into account the contribution of gluon operators (called twist-2 operators), see for details [58].

As shown by an effective non-relativistic field theory approach, the actual basis of operators that describe dark matter particles interacting with nuclei contains around 15 terms, and most of them do depend on the velocity or the momentum transfer [59-61]. When the dominant spin-independent and/or spin-dependent operators are zero, subdominant terms become relevant and might be in the sensitivity range of current experiments, see e.g. [62,63]. DARKSUSY has recently implemented the velocity dependence for the elastic scattering of dark matter.

SuPERIso Relic, MicrOMEGAs [64] and MADDM have recasted few experimental likelihoods or upper bounds to allow the user to directly compare the theoretical predictions with the most stringent experimental limits, such as XENON1T [65] for spin-independent and PICO-60 [66] for spin-dependent scattering. SuperIso Relic, MicrOMEGAs and DarkSUSY allow as well for modifications of the dark matter velocity distribution, to account for astrophysical uncertainties. 
Nuclear uncertainties are taken into account in all the tools by allowing the user to easily change the value of the nuclear form factors by hand.

There are also public codes devoted only to direct detection searches, such as DDCALC, included in DARKBIT [29], RAPIDD [67], which is based on the non-relativistic effective field theory description of dark matter interactions with nuclei, and RunDM [68]. The latter takes into account the running of the couplings from the UV scale of the model down to the keV-MeV scale of direct detection experiments.

Indirect detection Dark matter indirect searches look for WIMP annihilation (or decay) products in dense astrophysical environments, see e.g. [69, 70] for general reviews on the matter. In particularly, they look for an excess (with respect to the astrophysical background) in the flux of gamma rays, cosmic rays (positrons and anti-deuterium mainly) and neutrinos from the galactic centre, from dwarf spheroidal galaxies, from the Sun, namely everywhere the dark matter density is large and/or dominates over the baryonic component.

From the particle physics point of view, there are two key quantities relevant to obtain predictions for the expected flux: the velocity averaged annihilation cross-section $\langle\sigma v\rangle_{\text {ann }}$ and the energy spectrum $d N / d E$ of the final products listed above. The computation of the matrix element leading to $\langle\sigma v\rangle_{\text {ann }}$ proceeds in the same way as for the relic density in each tool, while the energy spectra are tabulated in all codes for two body final states. MADDMprovides the tabulated energy spectra, imported from PPPC4DMID [71], which effectively speeds up the computation of the expected flux, when the dark matter annihilates into the usual SM final states. Additionally MADDM allows the user to compute the energy spectra for any final state with $n$ particles, independently of their nature (SM or beyond), by automatically running Pyтнia 8 [72]. The energy spectra can change significantly if electroweak corrections are included, for which two treatments at the time of writing are available: PPPC4DMID, that is based on [73], and ref. [74]. Additionally, the shape of the energy spectrum can acquire spectral features if internal [75] or electroweak [76] Bremsstrahlung is considered ( $2 \rightarrow 3$ processes). The first is automatically included in MicrOMEGAs while both are encoded in DARKSUSY for specific models. MADDM can compute any of those corrections for a generic model if this is explicitly required by the user.

From the astrophysical point of view, indirect detection requires a lot of inputs and development. First, the energy spectrum is produced at the source; in case of charged particles, which diffuse in the galaxy, a propagation code is needed to obtain the correct spectrum at detection. Cosmic-ray propagation is available in all codes but MADDM, where instead an interface to the numerical code DRAGON [77] is provided. Gamma rays and neutrinos do not suffer propagation effects and point straight to the source where they were produced, hence their energy spectra is the same at source and detection point (modulo neutrino oscillations). Concerning the latter, one of the most promising searches is to look for neutrinos from the Sun and/or from the Earth [78] with IceCube [79]: these predictions are fully available in MicROMEGAs and DARKSUSY with a high level of precision. These predictions are rather involved as their require a modelling of the Sun and Earth together with the computation of neutrino oscillations in matter. Concerning gamma rays, one of the most sensitive search is the Fermi-LAT search from dwarf spheroidal galaxies [80]. For this search, MADDM and SUPERIso ReLIC not only compute the expected flux but provide as well a recasted Fermi-LAT likelihood analysis to compare directly the model parameter point with the experimental 
data. SuPerIso Relic in this respect is the public code that has the most advanced experimental module, as it provides as well a recasting of the AMS-02 antiproton analysis [81].

There are many public codes devoted to the study of astrophysical signals from dark matter, which are typically model-independent. Namely, they focus on the precise description of the astrophysical flux produced by dark matter and by the astrophysical background and they require the user input concerning the particle physics model. A non-exhaustive list is given by: GAMLike included in DARKBIt, GALPROP [82], DRAGON, PPPC4DMID, MADHAT [83], CLUMPY [84], $\chi$ aro $v$ [85] and USINE [86].

The output for relic density, direct detection and indirect detection of the different public codes has been compared in various configurations and with very different dark matter models. Overall, the results for the theoretical predictions generally agree very well, the discrepancies being never more than $10 \%$, which turns out to be of the order of the theoretical error. Sometimes, larger discrepancies are found, but usually, when this happens, the causes are well understood and trackable. For instance, in refs. [19, 39] the discrepancies in the relic density calculation arise in the different treatment of the QCD sector in MG5_AMC and CALCHeP. In summary, in the standard WIMP freeze-out scenario, all public codes have been deeply tested and compared, process that has allowed to solve numerous bugs.

\section{Tell me your model, I will tell you the tool}

The widening of the dark matter landscape has broaden the spectrum of theoretical predictions for relic density, direct and indirect detection and to a minor extent of LHC dark matter searches. In order to efficiently describe the phenomenology of the new avenues, the public tools are constantly evolving to encompass as much as possible the new predictions. I list below the remarkable features of each of the four numerical tools described in this review. While for WIMPs the various codes are more or less equivalent, it appears clear in the following that for the new avenues each one of them has definite characteristics that render it unique to the user.

\subsection{SuPERIso ReLIC: So far it's all about supersymmetry}

The current SuPERIso ReLIC version v4 is a mixed Fortran/C code which allows to compute comprehensively observables in supersymmetry, together with an experimental module that enables the user to automatically confront his/her model with experimental data for direct and indirect detection, as already described above. It is actually one of the reference codes to perform statistical comparisons between the SM and supersymmetric models in the light of recent collider, high energy, flavour physics and dark matter data, see e.g. [87].

Besides dealing with the supersymmetric WIMP phenomenology, SuPERIso Relic can be easily interfaced with ALTERBBN [88], a stand-alone public tool which allows to compute Big Bang Nucleosynthesis observables in standard and modified cosmologies. The combination of these two tools permits to test the supersymmetric dark matter hypothesis in alternative cosmologies, where for instance the presence of quintessence or scalar fields modifies the expansion rate of the universe and consequently the relic abundance of the neutralino, see [89]. 
Lastly, SuperIso Relic is foreseen to be able to compute dark matter observable for generic dark matter models in a near future. This augmented capacity is based on the public tool MARTY [90], a C++ framework that automates calculations from the Lagrangian to physical quantities such as matrix elements or cross-sections. This framework can fully simplify physical quantities in a large variety of models, making possible to quickly compare a dark matter model with all accessible experimental data.

\subsection{DARKSUSY: Towards dark sectors and new interactions}

The current DARKSUSY version (v6) is a Fortran code with a clear modular structure; in each module, such as the MSSM module, or the generic WIMP module, the user can select and extract the functions he/she needs and link them to his/her model by providing few inputs, such as the dark matter mass, $\langle\sigma v\rangle$, etc. DARKSUSY has implemented several features that allow the user to describe the phenomenology of generic dark sectors.

Concerning the relic density, besides the precision routines described above for the standard freeze-out case, it computes the freeze-out and the corresponding thermal $\langle\sigma v\rangle$ for dark matter particles in hidden sectors, which can have a temperature different than the photon temperature, see [91].

Dark matter in dark sectors can have large self-interactions, which can be obtained in two ways: (i) from strongly coupled dark sectors, similarly to QCD, or (ii) from weakly coupled theories featuring a very light mediating particle. The former case, where the large self-interactions do not depend on the dark matter velocity, is severely bounded from galaxy cluster observations, see e.g. the well-known constraints from the Bullet cluster [92]. In the latter case, self-interactions do depend on velocity and become stronger at smaller dark matter velocities. Phenomenologically, it is possible to have large effects on small scales consistent with the astrophysical constraints on larger scales. This is the case implemented in DARKSUSY [93], which includes the computation of the Sommerfeld enhancement (see e.g. [94-96] for its numerical solution), cutoff at small scales due to kinetic decoupling and the momentum transfer cross-section $\sigma_{T}$ not only in the Born limit but accounting for non-perturbative effects.

Concerning direct detection, DARKSUSY has started to cover the phenomenology of light dark matter, the so-called sub-GeV dark matter. It includes 'reverse' direct detection [97], where the dark matter is now the target and ordinary matter is the beam. More specifically, the number density of sub-GeV dark matter is very high, as its mass is small, hence the cosmic-ray propagation can be affected when these charged particles scatter elastically off dark matter particles in the galaxy. Building on top of this, one of the DARKSUSY authors has shown that current large volume direct detection and neutrino experiments are sensitive to a new population of dark matter particles, which is boosted at high velocity by up scattering off cosmic-rays close to the Sun position [98, 99]. The related theoretical predictions are available within the code. Lastly, notice that the interest in sub-GeV dark matter is spreading in the scientific community and other efforts are ongoing. For instance, a specific tool, Damascus [100], that simulates their scattering in underground detectors and the Earth overburden, is publicly available on the market. 


\subsection{MicrOMEGAs: The FIMP regime}

The current MicROMEGAs version v5.2 is a Fortran/C/C++ code which is fairly user-friendly: relic-density computations, direct detection and indirect detection predictions can all be switched on or off with simple flags in the main program. It is by far the most used tool in the dark matter community to study the phenomenology of WIMP generic models.

Besides the traditional $Z_{2}$ parity that is usually associated to dark matter particles (or $R$-parity in supersymmetry), the relic density computation has been extended to cover the case of a dark group with a $Z_{n}$ symmetry; this implies to add into the Boltzmann equation terms accounting for semi-annihilation processes [101], as well as the case of two DM candidates.

The most relevant novelty in MicrOMEGAs is the capability of computing the relic density of FIMPs via freeze-in. In short, the dark matter particles are too feebly interacting with SM particles in the early universe to be in chemical and kinetic equilibrium with the thermal bath. Dark matter is produced then either by the decay of an heavier (new) particles, either by the scattering of SM particles. Typically the initial dark matter number density is negligible, and it has been checked that the final value of the relic density is not very sensitive to this initial condition. The approach followed by MicROMEGAs tends to be as general as possible, namely based on only few assumptions, which should however be checked to be sure that they apply to the user model. MicROMEGAs considers three regimes for freeze-in: (i) dark matter production via the decay of a heavy mediator which is in thermal equilibrium, (ii) decay of a heavy mediator which is not in thermal equilibrium and (iii) $2 \rightarrow 2$ processes where SM particles produce two dark matter particles. It has been shown that the appropriate use of statistical distributions and thermal masses can lead to differences up to factors of two, hence those have been properly included in MicrOMEGAs. In addition, the FIMP phenomenology is relevant for LHC searches, as it can give rise to long lived particles [102], which can be considered using SModels [103].

\subsection{MADDM: Automatised annihilations for generic models}

The current MADDM version v3.1 is a mixed Python/Fortran code, which is based on the MG5_AMC platform and of which it inherits most features, such as the ability to compute any process automatically using the event generator MADEvent [104]. This software has the capability to compute any annihilation cross-section into $n$ final state particles at tree level order. Additionally, MADDM is capable to compute loop-induced processes, by definition leading order hence finite loop processes, for the dark matter. This ability is based on MADLoop [105]. A first attempt in the automatisation of loop-induced processes is presented in [19], however the code is currently being tested before its public release, which is foreseen for early 2021. The relevance of loop-induced processes is striking for dark matter observables: since the dark matter is neutral it can not couple directly to photons, hence the annihilation into di-photon can proceed only via loop (or effective vertex). Being a two body final state, each photon is monochromatic with an energy equal to the dark matter mass, since the annihilation occurs basically at rest. Such monochromatic lines, and in general sharp spectral features and edges in the gamma-ray energy spectra, are smoking gun for dark matter, as they can be hardly mimicked by astrophysical sources [106-108]. The other public codes can compute gamma-line predictions only for definite specific models, such as supersymmetry [107] 
or the inert doublet model [109], as all diagrams contributing to the cross-section are hard-coded. MADDM will be able to compute on the fly such predictions for a generic dark matter model.

Still concerning the gamma-ray phenomenology, an interesting possibility to test models of dark sector featuring long-lived mediator particles is to observe the gamma-ray emission from the Sun due to dark matter annihilation, which has been proposed by several authors [110-112] and has already evoked a search by the Fermi-LAT collaboration [113]. This module is not yet publicly available in MADDM but can be obtained by contacting the authors.

\section{Discussion and conclusions}

This review provides an (hopefully) unbiased overview of the status of the four major public tools for dark matter observables: SuperIso Relic, DARKSUSY, MicrOMEGAs and MAdDM. Concerning WIMPs, their phenomenology is very well covered in all of its necessary aspects; the user in this respect can simply choose the tool that better suits his/her needs and his/her model (files), knowing that the numerical discrepancies among tools are generically not larger than the percentage level. Concerning the new dark matter avenues, the dark sector phenomenology and sub-GeV dark matter particles begin to be pretty well described by DARKSUSY, while MicROMEGAs encompasses a fairly generic description of FIMPs. MADDM and SuPERIso Relic will soon give the possibility to compute loop (induced) processes automatically within generic dark matter extensions of the SM. Last but not least, numerical tools are nowadays user friendly in both their usage and in their portability towards other tools for high energy physics. The use of FeynRules, MadDM, MicrOMEGAs, MG5_AMC, MadAnalysis 5, linked one after the other, has become a common practice in phenomenological papers, as well as the accomplishment of global fits is taking more and more momentum thanks to the modular tools developed by the GAMBIT collaboration. Typically, the confrontation of theoretical predictions with experimental data stands at the end of the chain for global analyses. A correct and sophisticated way of performing this comparison entails a likelihood analysis, as this confronts directly the model with the data. The exclusion bounds hence do not rely on approximations and assumptions that might not be verified for that particular model. The key ingredient is the experimental likelihood function: many efforts from the theoretical side is concentrated to reconstruct experimental likelihoods in order to recast properly limits from the collaborations. Usually, this is not a straightforward procedure when the data and the experimental details are not public. In this respect, in the past few years there has been a real improvement in the interactions between the experimental and theoretical communities and many data and likelihood functions have become public, such as for the Fermi-LAT dwarf spheroidal one. There are sectors where this is not yet common practice, but the efforts and synergies are moving to the good direction for data reinterpretation, see e.g. [114].

Overall, this review has an optimistic view of the dark matter tool landscape. Nevertheless, this is not the end of the story and the public codes are continuously debugged, ameliorated and improved with new physics, as the quest for dark matter is not yet over. This calls for the continuous development of a platform for comprehensive studies and for the identification of new directions for the experimental searches to look for dark matter signatures. 


\section{Acknowledgments}

The author warmly acknowledges the organisers of TOOLS2020 for the invitation to give this review talk, which preparation has been enriching. The author is supported by the Innoviris ATTRACT 2018104 BECAP 2 agreement.

\section{References}

[1] Planck collaboration, N. Aghanim et al., Planck 2018 results. VI. Cosmological parameters, Astron. Astrophys. 641 (2020) A6, [1807.06209].

[2] J. F. Navarro, A. Ludlow, V. Springel, J. Wang, M. Vogelsberger, S. D. White et al., The Diversity and Similarity of Cold Dark Matter Halos, Mon. Not. Roy. Astron. Soc. 402 (2010) 21, [0810.1522].

[3] M. Boylan-Kolchin, V. Springel, S. D. White, A. Jenkins and G. Lemson, Resolving Cosmic Structure Formation with the Millennium-II Simulation, Mon. Not. Roy. Astron. Soc. 398 (2009) 1150, [0903.3041].

[4] M. Vogelsberger, S. Genel, V. Springel, P. Torrey, D. Sijacki, D. Xu et al., Introducing the Illustris Project: Simulating the coevolution of dark and visible matter in the Universe, Mon. Not. Roy. Astron. Soc. 444 (2014) 1518-1547, [1405.2921].

[5] J. Bovy and S. Tremaine, On the local dark matter density, Astrophys. J. 756 (2012) 89, [1205.4033].

[6] J. Silk et al., Particle Dark Matter: Observations, Models and Searches. Cambridge Univ. Press, Cambridge, 2010, 10.1017/CBO9780511770739.

[7] R. K. Leane, T. R. Slatyer, J. F. Beacom and K. C. Ng, GeV-scale thermal WIMPs: Not even slightly ruled out, Phys. Rev. D 98 (2018) 023016, [1805. 10305].

[8] N. Bernal, M. Heikinheimo, T. Tenkanen, K. Tuominen and V. Vaskonen, The Dawn of FIMP Dark Matter: A Review of Models and Constraints, Int. J. Mod. Phys. A 32 (2017) 1730023, [1706.07442].

[9] M. Battaglieri et al., US Cosmic Visions: New Ideas in Dark Matter 2017: Community Report, in U.S. Cosmic Visions: New Ideas in Dark Matter, 7, 2017. 1707.04591.

[10] J. McDonald, Thermally generated gauge singlet scalars as selfinteracting dark matter, Phys. Rev. Lett. 88 (2002) 091304, [hep-ph/0106249].

[11] L. J. Hall, K. Jedamzik, J. March-Russell and S. M. West, Freeze-In Production of FIMP Dark Matter, JHEP 03 (2010) 080, [0911. 1120].

[12] A. Alloul, N. D. Christensen, C. Degrande, C. Duhr and B. Fuks, FeynRules 2.0 - A complete toolbox for tree-level phenomenology, Comput. Phys. Commun. 185 (2014) 2250-2300, [1310.1921]. 
[13] F. Staub, SARAH 3.2: Dirac Gauginos, UFO output, and more, Comput. Phys. Commun. 184 (2013) 1792-1809, [1207.0906].

[14] A. Semenov, LanHEP - A package for automatic generation of Feynman rules from the Lagrangian. Version 3.2, Comput. Phys. Commun. 201 (2016) 167-170, [1412.5016].

[15] D. Abercrombie et al., Dark Matter Benchmark Models for Early LHC Run-2 Searches: Report of the ATLAS/CMS Dark Matter Forum, Phys. Dark Univ. 27 (2020) 100371, [1507.00966].

[16] M. Backovic̀, M. Krämer, F. Maltoni, A. Martini, K. Mawatari and M. Pellen, Higher-order QCD predictions for dark matter production at the LHC in simplified models with s-channel mediators, Eur. Phys. J. C75 (2015) 482, [1508.05327].

[17] G. Busoni et al., Recommendations on presenting LHC searches for missing transverse energy signals using simplified s-channel models of dark matter, Phys. Dark Univ. 27 (2020) 100365, [1603.04156].

[18] C. Arina, Impact of cosmological and astrophysical constraints on dark matter simplified models, Front. Astron. Space Sci. 5 (2018) 30, [1805.04290].

[19] C. Arina, B. Fuks and L. Mantani, A universal framework for t-channel dark matter models, Eur. Phys. J. C 80 (2020) 409, [2001.05024].

[20] J. Alwall, M. Herquet, F. Maltoni, O. Mattelaer and T. Stelzer, MadGraph 5 : Going Beyond, JHEP 06 (2011) 128, [1106.0522].

[21] C. Degrande, C. Duhr, B. Fuks, D. Grellscheid, O. Mattelaer and T. Reiter, UFO - The Universal FeynRules Output, Comput. Phys. Commun. 183 (2012) 1201-1214, [1108.2040].

[22] E. Conte, B. Fuks and G. Serret, MadAnalysis 5, A User-Friendly Framework for Collider Phenomenology, Comput. Phys. Commun. 184 (2013) 222-256, [1206. 1599].

[23] F. Ambrogi, S. Kraml, S. Kulkarni, U. Laa, A. Lessa, V. Magerl et al., SModelS v1.1 user manual: Improving simplified model constraints with efficiency maps, Comput. Phys. Commun. 227 (2018) 72-98, [1701.06586].

[24] C. Arina et al., A comprehensive approach to dark matter studies: exploration of simplified top-philic models, JHEP 11 (2016) 111, [1605.09242].

[25] D. Barducci, G. Belanger, J. Bernon, F. Boudjema, J. Da Silva, S. Kraml et al., Collider limits on new physics within micrOMEGAs_4.3, Comput. Phys. Commun. 222 (2018) 327-338, [1606.03834].

[26] C. Arina, B. Fuks, L. Mantani, H. Mies, L. Panizzi and J. Salko, Closing in on t-channel simplified dark matter models, 2010.07559. 
[27] M. D. Goodsell, S. Kraml, H. Reyes-González and S. L. Williamson, Constraining Electroweakinos in the Minimal Dirac Gaugino Model, SciPost Phys. 9 (2020) 047, [2007.08498].

[28] GAMBIT collaboration, P. Athron et al., GAMBIT: The Global and Modular Beyond-the-Standard-Model Inference Tool, Eur. Phys. J. C 77 (2017) 784, [1705.07908].

[29] GAMBIT Dark Matter Workgroup collaboration, T. Bringmann et al., DarkBit: A GAMBIT module for computing dark matter observables and likelihoods, Eur. Phys. J. C 77 (2017) 831, [1705.07920].

[30] GAMBIT Cosmology Workgroup collaboration, J. J. Renk et al., CosmoBit: A GAMBIT module for computing cosmological observables and likelihoods, 2009.03286.

[31] GAMBIT collaboration, G. D. Martinez, J. McKay, B. Farmer, P. Scott, E. Roebber, A. Putze et al., Comparison of statistical sampling methods with ScannerBit, the GAMBIT scanning module, Eur. Phys. J. C 77 (2017) 761, [1705.07959].

[32] GAMBIT collaboration, P. Athron et al., Global fits of GUT-scale SUSY models with GAMBIT, Eur. Phys. J. C 77 (2017) 824, [1705.07935].

[33] A. Kvellestad, P. Scott and M. White, GAMBIT and its Application in the Search for Physics Beyond the Standard Model, 1912.04079.

[34] P. Athron et al., Global fits of axion-like particles to XENONIT and astrophysical data, 2007.05517.

[35] C. Arina, A. Beniwal, C. Degrande, J. Heisig and A. Scaffidi, Global fit of pseudo-Nambu-Goldstone Dark Matter, JHEP 04 (2020) 015, [1912 . 04008].

[36] A. Arbey, F. Mahmoudi and G. Robbins, SuperIso Relic v4: A program for calculating dark matter and flavour physics observables in Supersymmetry, Comput. Phys. Commun. 239 (2019) 238-264, [1806.11489].

[37] T. Bringmann, J. Edsjö, P. Gondolo, P. Ullio and L. Bergström, DarkSUSY 6 : An Advanced Tool to Compute Dark Matter Properties Numerically, JCAP 07 (2018) 033, [1802 . 03399].

[38] G. Bélanger, F. Boudjema, A. Goudelis, A. Pukhov and B. Zaldivar, micrOMEGAs5.0 : Freeze-in, Comput. Phys. Commun. 231 (2018) 173-186, [1801.03509].

[39] F. Ambrogi, C. Arina, M. Backovic, J. Heisig, F. Maltoni, L. Mantani et al., MadDM v.3.0: a Comprehensive Tool for Dark Matter Studies, Phys. Dark Univ. 24 (2019) 100249, [1804.00044].

[40] C. Arina, J. Heisig, F. Maltoni, L. Mantani, D. Massaro, O. Mattelaer et al., Studying dark matter with MadDM 3.1: a short user guide, 12, 2020. 2012.09016. 
[41] G. Belanger, F. Boudjema, A. Pukhov and A. Semenov, MicrOMEGAs: A Program for calculating the relic density in the MSSM, Comput. Phys. Commun. 149 (2002) 103-120, [hep-ph/0112278].

[42] P. Gondolo, J. Edsjo, P. Ullio, L. Bergstrom, M. Schelke and E. A. Baltz, DarkSUSY: Computing supersymmetric dark matter properties numerically, JCAP 0407 (2004) 008, [astro-ph/0406204].

[43] A. Arbey and F. Mahmoudi, SuperIso Relic: A Program for calculating relic density and flavor physics observables in Supersymmetry, Comput. Phys. Commun. 181 (2010) 1277-1292, [0906.0369].

[44] M. Backovic̀, K. Kong and M. McCaskey, MadDM v.1.0: Computation of Dark Matter Relic Abundance Using MadGraph5, Phys. Dark Univ. 5-6 (2014) 18-28, [1308.4955].

[45] P. Gondolo and G. Gelmini, Cosmic abundances of stable particles: Improved analysis, Nucl. Phys. B 360 (1991) 145-179.

[46] J. Edsjo and P. Gondolo, Neutralino relic density including coannihilations, Phys. Rev. D 56 (1997) 1879-1894, [hep-ph/9704361].

[47] G. Steigman, B. Dasgupta and J. F. Beacom, Precise Relic WIMP Abundance and its Impact on Searches for Dark Matter Annihilation, Phys. Rev. D 86 (2012) 023506, [1204 . 3622].

[48] A. Belyaev, N. D. Christensen and A. Pukhov, CalcHEP 3.4 for collider physics within and beyond the Standard Model, Comput. Phys. Commun. 184 (2013) 1729-1769, [1207.6082].

[49] F. Giacchino, A. Ibarra, L. Lopez Honorez, M. H. G. Tytgat and S. Wild, Signatures from Scalar Dark Matter with a Vector-like Quark Mediator, JCAP 1602 (2016) 002, [1511.04452].

[50] M. Beneke, A. Bharucha, F. Dighera, C. Hellmann, A. Hryczuk, S. Recksiegel et al., Relic density of wino-like dark matter in the MSSM, JHEP 03 (2016) 119, [1601.04718].

[51] B. Herrmann and M. Klasen, SUSY-QCD Corrections to Dark Matter Annihilation in the Higgs Funnel, Phys. Rev. D 76 (2007) 117704, [0709.0043].

[52] T. Bringmann and S. Hofmann, Thermal decoupling of WIMPs from first principles, JCAP 04 (2007) 016, [hep-ph/0612238].

[53] T. Binder, T. Bringmann, M. Gustafsson and A. Hryczuk, Early kinetic decoupling of dark matter: when the standard way of calculating the thermal relic density fails, Phys. Rev. D 96 (2017) 115010, [1706.07433].

[54] A. Gould, J. A. Frieman and K. Freese, Probing the Earth With Wimps, Phys. Rev. D 39 (1989) 1029.

[55] D. G. Cerdeno and A. M. Green, Direct detection of WIMPs, 1002. 1912. 
[56] C. Arina, Bayesian analysis of multiple direct detection experiments, Phys. Dark Univ. 5-6 (2014) 1-17, [1310.5718].

[57] M. Schumann, Direct Detection of WIMP Dark Matter: Concepts and Status, J. Phys. G 46 (2019) 103003, [1903.03026].

[58] J. Hisano, R. Nagai and N. Nagata, Effective Theories for Dark Matter Nucleon Scattering, JHEP 05 (2015) 037, [1502.02244].

[59] J. Fan, M. Reece and L.-T. Wang, Non-relativistic effective theory of dark matter direct detection, JCAP 11 (2010) 042, [1008.1591].

[60] V. Cirigliano, M. L. Graesser and G. Ovanesyan, WIMP-nucleus scattering in chiral effective theory, JHEP 10 (2012) 025, [1205. 2695].

[61] A. L. Fitzpatrick, W. Haxton, E. Katz, N. Lubbers and Y. Xu, The Effective Field Theory of Dark Matter Direct Detection, JCAP 1302 (2013) 004, [1203. 3542].

[62] C. Arina, E. Del Nobile and P. Panci, Dark Matter with Pseudoscalar-Mediated Interactions Explains the DAMA Signal and the Galactic Center Excess, Phys. Rev. Lett. 114 (2015) 011301, [1406.5542].

[63] N. Bozorgnia, D. G. Cerdeño, A. Cheek and B. Penning, Opening the energy window on direct dark matter detection, JCAP 12 (2018) 013, [1810.05576].

[64] G. Belanger, A. Mjallal and A. Pukhov, Recasting direct detection limits within micrOMEGAs and implication for non-standard Dark Matter scenarios, 2003.08621.

[65] XENON collaboration, E. Aprile et al., Dark Matter Search Results from a One Ton-Year Exposure of XENON1T, Phys. Rev. Lett. 121 (2018) 111302, [1805.12562].

[66] PICO collaboration, C. Amole et al., Dark Matter Search Results from the PICO-60 $C_{3} F_{8}$ Bubble Chamber, Phys. Rev. Lett. 118 (2017) 251301, [1702.07666].

[67] D. Cerdeño, A. Cheek, E. Reid and H. Schulz, Surrogate Models for Direct Dark Matter Detection, JCAP 08 (2018) 011, [1802.03174].

[68] F. D'Eramo, B. J. Kavanagh and P. Panci, You can hide but you have to run: direct detection with vector mediators, JHEP 08 (2016) 111, [1605.04917].

[69] M. Cirelli, Indirect Searches for Dark Matter: a status review, Pramana 79 (2012) 1021-1043, [1202. 1454].

[70] J. M. Gaskins, A review of indirect searches for particle dark matter, Contemp. Phys. 57 (2016) 496-525, [1604.00014].

[71] M. Cirelli, G. Corcella, A. Hektor, G. Hutsi, M. Kadastik, P. Panci et al., PPPC 4 DM ID: A Poor Particle Physicist Cookbook for Dark Matter Indirect Detection, JCAP 1103 (2011) 051, [1012.4515]. 
[72] T. Sjostrand, S. Ask, J. R. Christiansen, R. Corke, N. Desai, P. Ilten et al., An Introduction to PYTHIA 8.2, Comput. Phys. Commun. 191 (2015) 159-177, [1410.3012].

[73] P. Ciafaloni, D. Comelli, A. Riotto, F. Sala, A. Strumia and A. Urbano, Weak Corrections are Relevant for Dark Matter Indirect Detection, JCAP 1103 (2011) 019, [1009.0224].

[74] C. W. Bauer, N. L. Rodd and B. R. Webber, Dark Matter Spectra from the Electroweak to the Planck Scale, 2007.15001.

[75] T. Bringmann and C. Weniger, Gamma Ray Signals from Dark Matter: Concepts, Status and Prospects, Phys. Dark Univ. 1 (2012) 194-217, [1208. 5481].

[76] T. Bringmann and F. Calore, Significant Enhancement of Neutralino Dark Matter Annihilation from Electroweak Bremsstrahlung, Phys. Rev. Lett. 112 (2014) 071301, [1308. 1089].

[77] C. Evoli, D. Gaggero, D. Grasso and L. Maccione, Cosmic-Ray Nuclei, Antiprotons and Gamma-rays in the Galaxy: a New Diffusion Model, JCAP 0810 (2008) 018, [0807. 4730].

[78] A. Gould, Resonant Enhancements in WIMP Capture by the Earth, Astrophys. J. 321 (1987) 571.

[79] IceCube collaboration, M. G. Aartsen et al., Search for annihilating dark matter in the Sun with 3 years of IceCube data, Eur. Phys. J. C77 (2017) 146, [1612 . 05949].

[80] DES, Fermi-LAT collaboration, A. Albert et al., Searching for Dark Matter Annihilation in Recently Discovered Milky Way Satellites with Fermi-LAT, Astrophys. J. 834 (2017) 110, [1611.03184].

[81] AMS Collaboration collaboration, M. Aguilar et al., Antiproton flux, antiproton-to-proton flux ratio, and properties of elementary particle fluxes in primary cosmic rays measured with the alpha magnetic spectrometer on the international space station, Phys. Rev. Lett. 117 (Aug, 2016) 091103.

[82] A. E. Vladimirov, S. W. Digel, G. Johannesson, P. F. Michelson, I. V. Moskalenko, P. L. Nolan et al., GALPROP WebRun: an internet-based service for calculating galactic cosmic ray propagation and associated photon emissions, Comput. Phys. Commun. 182 (2011) 1156-1161, [1008.3642].

[83] K. K. Boddy, S. Hill, J. Kumar, P. Sandick and B. Shams Es Haghi, MADHAT: Model-Agnostic Dark Halo Analysis Tool, 1910.02890.

[84] V. Bonnivard, M. Hütten, E. Nezri, A. Charbonnier, C. Combet and D. Maurin, CLUMPY : Jeans analysis, $\gamma$-ray and $v$ fluxes from dark matter (sub-)structures, Comput. Phys. Commun. 200 (2016) 336-349, [1506. 07628].

[85] Q. Liu, J. Lazar, C. A. Argüelles and A. Kheirandish, yarov: a tool for neutrino flux generation from WIMPs, JCAP 10 (2020) 043, [2007. 15010]. 
[86] D. Maurin, USINE: semi-analytical models for Galactic cosmic-ray propagation, Comput. Phys. Commun. 247 (2020) 106942, [1807. 02968].

[87] A. Hryczuk, K. Jodlowski, E. Moulin, L. Rinchiuso, L. Roszkowski, E. M. Sessolo et al., Testing dark matter with Cherenkov light - prospects of H.E.S.S. and CTA for exploring minimal supersymmetry, JHEP 10 (2019) 043, [1905.00315].

[88] A. Arbey, J. Auffinger, K. Hickerson and E. Jenssen, AlterBBN v2: A public code for calculating Big-Bang nucleosynthesis constraints in alternative cosmologies, Comput. Phys. Commun. 248 (2020) 106982, [1806.11095].

[89] A. Arbey, J. Ellis, F. Mahmoudi and G. Robbins, Dark Matter Casts Light on the Early Universe, JHEP 10 (2018) 132, [1807.00554].

[90] G. Uhlrich, F. Mahmoudi and A. Arbey, MARTY - Modern ARtificial Theoretical phYsicist: A C ++ framework automating symbolic calculations Beyond the Standard Model, 2011.02478.

[91] T. Bringmann, P. F. Depta, M. Hufnagel and K. Schmidt-Hoberg, Precise dark matter relic abundance in decoupled sectors, 2007.03696.

[92] M. Markevitch, A. Gonzalez, D. Clowe, A. Vikhlinin, L. David, W. Forman et al., Direct constraints on the dark matter self-interaction cross-section from the merging galaxy cluster 1E0657-56, Astrophys. J. 606 (2004) 819-824, [astro-ph/0309303].

[93] T. Bringmann, F. Kahlhoefer, K. Schmidt-Hoberg and P. Walia, Strong constraints on self-interacting dark matter with light mediators, Phys. Rev. Lett. 118 (2017) 141802, [1612.00845].

[94] R. Iengo, Sommerfeld enhancement for a Yukawa potential, 0903.0317.

[95] R. Iengo, Sommerfeld enhancement: General results from field theory diagrams, JHEP 05 (2009) 024, [0902.0688].

[96] C. Arina, F.-X. Josse-Michaux and N. Sahu, Constraining Sommerfeld Enhanced Annihilation Cross-sections of Dark Matter via Direct Searches, Phys. Lett. B 691 (2010) 219-224, [1004.0645].

[97] C. V. Cappiello, K. C. Ng and J. F. Beacom, Reverse Direct Detection: Cosmic Ray Scattering With Light Dark Matter, Phys. Rev. D 99 (2019) 063004, [1810 . 07705].

[98] T. Bringmann and M. Pospelov, Novel direct detection constraints on light dark matter, Phys. Rev. Lett. 122 (2019) 171801, [1810. 10543].

[99] R. Plestid, V. Takhistov, Y.-D. Tsai, T. Bringmann, A. Kusenko and M. Pospelov, New Constraints on Millicharged Particles from Cosmic-ray Production, 2002 . 11732.

[100] T. Emken and C. Kouvaris, DaMaSCUS: The Impact of Underground Scatterings on Direct Detection of Light Dark Matter, JCAP 10 (2017) 031, [1706. 02249]. 
[101] G. Bélanger, K. Kannike, A. Pukhov and M. Raidal, Minimal semi-annihilating $\mathbb{Z}_{N}$ scalar dark matter, JCAP 06 (2014) 021, [1403.4960].

[102] J. Alimena et al., Searching for long-lived particles beyond the Standard Model at the Large Hadron Collider, J. Phys. G 47 (2020) 090501, [1903. 04497].

[103] F. Ambrogi et al., SModelS v1.2: long-lived particles, combination of signal regions, and other novelties, Comput. Phys. Commun. 251 (2020) 106848, [1811.10624].

[104] F. Maltoni and T. Stelzer, MadEvent: Automatic event generation with MadGraph, JHEP 02 (2003) 027, [hep-ph/0208156].

[105] V. Hirschi, R. Frederix, S. Frixione, M. V. Garzelli, F. Maltoni and R. Pittau, Automation of one-loop QCD corrections, JHEP 05 (2011) 044, [1103.0621].

[106] A. Bouquet, P. Salati and J. Silk, Gamma-Ray Lines as a Probe for a Cold Dark Matter Halo, Phys. Rev. D40 (1989) 3168.

[107] L. Bergstrom, Radiative Processes in Dark Matter Photino Annihilation, Phys. Lett. B225 (1989) 372-380.

[108] S. Rudaz, On the Annihilation of Heavy Neutral Fermion Pairs Into Monochromatic gamma-rays and Its Astrophysical Implications, Phys. Rev. D39 (1989) 3549.

[109] M. Gustafsson, E. Lundstrom, L. Bergstrom and J. Edsjo, Significant Gamma Lines from Inert Higgs Dark Matter, Phys. Rev. Lett. 99 (2007) 041301, [astro-ph/0703512].

[110] B. Batell, M. Pospelov, A. Ritz and Y. Shang, Solar Gamma Rays Powered by Secluded Dark Matter, Phys. Rev. D 81 (2010) 075004, [0910.1567].

[111] R. K. Leane, K. C. Y. Ng and J. F. Beacom, Powerful Solar Signatures of Long-Lived Dark Mediators, Phys. Rev. D 95 (2017) 123016, [1703.04629].

[112] C. Arina, M. Backović, J. Heisig and M. Lucente, Solar y rays as a complementary probe of dark matter, Phys. Rev. D 96 (2017) 063010, [1703.08087].

[113] M. Mazziotta, F. Loparco, D. Serini, A. Cuoco, P. De La Torre Luque, F. Gargano et al., Search for dark matter signatures in the gamma-ray emission towards the Sun with the Fermi Large Area Telescope, Phys. Rev. D 102 (2020) 022003, [2006.04114].

[114] LHC Reinterpretation Forum collaboration, W. Abdallah et al., Reinterpretation of LHC Results for New Physics: Status and Recommendations after Run 2, SciPost Phys. 9 (2020) 022, [2003.07868]. 